\title{
Use of the Heartrail II catheter as a distal stent delivery device; an extended case series.
}

Abstract

AIMS:

The Terumo Heartrail catheter (Terumo Corp., Tokyo, Japan) allows extra deep catheter intubation of coronary vessels and has been shown to be useful in CTO lesions. The aim of this study is to assess the safety and efficacy of using the Heartrail II catheter as a distal stent delivery system in PCI following failure of conventional techniques.

\section{METHODS AND RESULTS:}

We prospectively identified cases performed over a 15-month period in which a Heartrail catheter was used to facilitate stent delivery following failure of conventional techniques. Stent delivery using the Heartrail catheter was performed in 35 cases and was successful in 31 cases. Success rates of $100 \%$ in grafts, $95 \%$ in RCA, $80 \%$ in LAD and $60 \%$ in circumflex cases were recorded respectively. Successful stent delivery was associated with intubation depth, with 29/29 succeeding when the intubation depth was $>2 \mathrm{~cm}$ and failure in 4/5 cases when the intubation depth $<$ or $=2 \mathrm{~cm}$. There were no complications related to deep intubation of the catheter.

\section{CONCLUSIONS:}

Use of the Heartrail catheter is safe and highly effective for aiding stent delivery across proximal obstructions in both left and right coronary systems. The small number of unsuccessful cases were related to inability of the catheter to traverse stenotic proximal obstructions within $2 \mathrm{~cm}$ of the RCA and LCA origins. 